8th Alexander Friedmann International Seminar

on Gravitation and Cosmology

International Journal of Modern Physics: Conference Series

Vol. 3 (2011) 246-253

(C) World Scientific Publishing Company

DOI: $10.1142 /$ S2010194511001334

\title{
THE DEGENERACY PROBLEM IN LAMBDA-CDM COSMOLOGICAL MODELS
}

\author{
ARMANDO BERNUI \\ ICE - Universidade Federal de Itajubá \\ Itajubá - MG, Brazil \\ abernui@gmail.com
}

Received 11 June 2011

Revised 18 July 2011

\begin{abstract}
Recent measurements of the cosmic microwave background radiation (CMB) from the WMAP satellite led to formulate a successful concordance cosmological model, termed $\Lambda$ CDM. This model satisfactorily explains the origin and structure of the CMB temperature fluctuations, from small to large angular scales, and moreover it accurately fits - with only six parameters- the CMB angular power spectrum. Despite of their triumphs in describing the observed WMAP data, we notice that some $\Lambda$ CDM cosmological parameters can attain, due to their error bars, slightly different values and this degree of freedom could produce a significant impact in our understanding of the primordial universe. We are talking about the degeneracy problem, that is cosmological models with parameters that are a little bit different from those given by the $\Lambda$ CDM model but fits equally well the angular power spectrum of the CMB data. Our interest here is to investigate the Gaussian statistical property, at large angular scales, in two sets of Monte Carlo CMB maps produced by seeding them with slightly different $\Lambda$ CDM angular power spectra.
\end{abstract}

Keywords: Cosmology; early universe; cosmic microwave background.

PACS numbers: 98.80.-k, 98.70.Vc, 98.80.Es, 98.80.Cq

\section{Introduction}

Successive data releases from the Wilkinson Microwave Anisotropy Probe (WMAP $)^{1}$ have confirmed the validity of the concordance cosmological model, best known as $\Lambda \mathrm{CDM}$. The model is obtained through the best-fitting of the cosmic microwave background radiation (CMB) angular power spectrum, as a result it provides the best values for the cosmological parameters, which of course are not sharp values but values within small intervals due to the error and uncertainty limits. For this, it is interesting to investigate synthetic CMB maps obtained from angular power spectra generated by very similar (but not equal) cosmological parameters that fit the WMAP data as well as the $\Lambda$ CDM model. One such parameter is the spectral index of primordial fluctuations $n_{s}$. The main difficulty to establish the correct spectral index value is that it determines the form of the angular power spectra 
at the largest scales (i.e. low multipoles), precisely the region where the cosmic variance uncertainty is large and dominates other effects.

The importance to determine, with the highest degree of accuracy, the value of the spectral index $n_{s}$ is because it is related to physical processes of the primordial universe (remember that the CMB radiation is -with present techniques- the oldest cosmological observable). And because such processes probably left imprints in the statistical attributes of the observed CMB, it deserves a meticulous analysis the relationship between the spectral index and the Gaussian properties of the CMB temperature field. For instance, the value $n_{s}=1$ means that the CMB temperature fluctuations correspond to a statistically Gaussian random field, therefore small variations from this value should be correlated with small deviations of Gaussianity in the data.

For these reasons, besides the determination of the spectral index $n_{s}$ by fitting the CMB angular power spectrum, the released data from the $\mathrm{WMAP}^{1}$ is nowadays under rigorous scrutiny for the possible deviations from Gaussianity ${ }^{2-7}$ and from statistical isotropy ${ }^{8-18}$ in the CMB data, properties that could be, in some sense, related. Moreover, a detection or non-detection of primordial non-Gaussianity in the $\mathrm{CMB}$ data is crucial to discriminate among inflationary models, and to test alternative scenarios of the early universe. ${ }^{19}$

We shall investigate the effects caused on the statistical properties of two sets of Monte Carlo CMB maps produced by seeding them with slightly different angular power spectra, each one obtained using the same cosmological parameters as the $\Lambda$ CDM model except that we consider different spectral indexes values: $n_{s}=$ 0.96 and 1.00, respectively. To detect the presence of Gaussian deviations in these CMB maps we use two recently published non-Gaussian indicators, ${ }^{20}$ based on the application of skewness and kurtosis in large-angle patches of CMB temperature maps, which allow us to construct the $\mathbf{S}$ - and $\mathbf{K}$-maps (for details see Sec. 3 ) from which we calculate their angular power spectra (APS). After that, we compare the APS of the two sets of $\mathbf{S}$-maps obtained by applying our skewness tool to both sets of Monte Carlo CMB maps. Similar procedures are done with the two sets of $\mathbf{K}$-maps. Our results show that, in the mean, the Gaussianity property of these sets of Monte Carlo maps is different, and this fact seems to be crucial when one has to quantify the confidence level of some data analysis result. That is to say, the statistical-significance evaluation of a result concerning a WMAP CMB map is model dependent.

This work is organized as follows. In Sec. 2 we explain how to produce the sets of simulated Monte Carlo CMB maps based on the $\Lambda$ CDM angular power spectrum, and also the ILC WMAP map, used in the analyses. In Sec. 3 we describe our method to analyze the statistical properties of the CMB maps. Finally, in Sec. 4 we summarize our results and formulate our conclusions and perspectives for future works. 


\section{2. $\Lambda \mathrm{CDM}$ Angular Power Spectra and Monte Carlo CMB data}

The degeneracy problem in the concordance cosmological model consists on having two or more APS similar to that one of the $\Lambda$ CDM model obtained with slightly different cosmological parameter value(s), but all of them consistent with current data because of the error bars.

Here we shall study two $\Lambda$ CDM APS with the spectral indexes attaining slightly different values: $n_{s}=0.96,1.00$. For this, we use the CMBFAst tool ${ }^{22}$ to generate the corresponding APS seeds that are then used in the SYNFAST algorithm ${ }^{23}$ to produce two sets of 1000 random realizations each. As we show in Fig. 1, left panel, the $\Lambda$ CDM APS corresponding to $n_{s}=0.96$ is very similar to the APS obtained with the same cosmological parameters as the concordance model, except that $n_{s}=1.00$. Moreover, we also observe in the right panel that both APS fits suitably the APS from WMAP data. The comparison of the statistical properties between these two sets of Monte Carlo CMB maps constitute the target of our analysis.

It is well known that foregrounds introduce non-Gaussianities in the CMB data. For these the WMAP science team have performed substantial efforts to minimize the presence of foregrounds in the measured CMB data. The recently released sevenyear foreground-reduced ILC map represents well a CMB map that is consistent with Gaussianity, after the application of the KQ75-7yr mask. ${ }^{1}$ We find interesting to use this WMAP map to test the mean statistical properties of these two sets of Monte Carlo CMB maps.

In the next section we shall describe our method to analyze the statistical features of the CMB maps, both Monte Carlo and WMAP ones. The comparison of the statistical properties between these two sets of Monte Carlo CMB maps, together with the analysis of the ILC map, will be performed in the last section.

\section{The Method for Statistical Analysis}

In this section we briefly present how to construct two non-Gaussinity indicators. For a detailed discussion of these indicators we refer the readers to Ref. 20.

Consider a discrete set of points $\left\{j=1, \ldots, N_{\mathrm{c}}\right\}$ uniformly distributed on the sphere $S^{2}$ as the center of spherical caps of a given aperture $\gamma$, and calculate for each cap $j$ the skewness and kurtosis values

$$
S_{j} \equiv \frac{1}{N_{\mathrm{p}} \sigma_{j}^{3}} \sum_{i=1}^{N_{\mathrm{p}}}\left(T_{i}-\bar{T}_{j}\right)^{3}, \quad K_{j} \equiv \frac{1}{N_{\mathrm{p}} \sigma_{j}^{4}} \sum_{i=1}^{N_{\mathrm{p}}}\left(T_{i}-\bar{T}_{j}\right)^{4}-3,
$$

where $N_{\mathrm{p}}$ is the number of pixels in the $j^{\text {th }}$ cap, $T_{i}$ is the temperature at the $i^{\text {th }}$ pixel, $\bar{T}_{j}$ is the CMB mean temperature on the $j^{\text {th }}$ cap, and $\sigma_{j}$ is the standard deviation for each $j$. The sets of values $\left\{S_{j}, j=1, \ldots, N_{\mathrm{c}}\right\}$ and $\left\{K_{j}, j=1, \ldots, N_{\mathrm{c}}\right\}$ can then be viewed as a measure of the non-Gaussianity in the direction $\left(\theta_{j}, \phi_{j}\right)$ (which corresponds to the center of the $j^{\text {th }}$ cap). Patching together the $\left\{S_{j}\right\}$ and $\left\{K_{j}\right\}$ values, we obtain the functions $S=S(\theta, \phi)$ and $K=K(\theta, \phi)$ defined on 

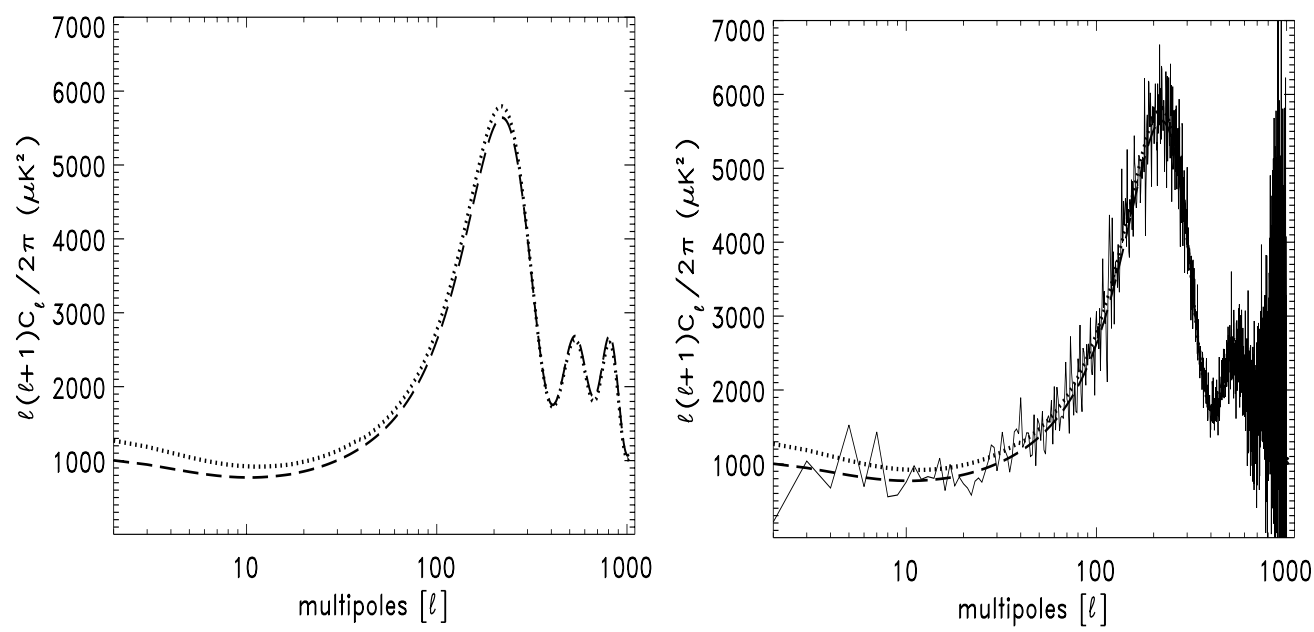

Fig. 1. (Left panel) Angular power spectra for $\Lambda$ CDM models with slightly different values for the spectral index: $n_{s}=0.96$ (dotted curve) and $n_{s}=1.00$ (dashed curve). (Right panel) The same angular power spectra appearing in the left panel plus the angular power spectrum from WMAP-7yr data (continuous noisy curve).

$S^{2}$. Throughout this work the sky map representation of the functions $S=S(\theta, \phi)$ and $K=K(\theta, \phi)$ are named, respectively, $\mathbf{S}$-map and $\mathbf{K}$-map. Clearly, one can expand each of these functions in their spherical harmonics and calculate their corresponding angular power spectrum. For the skewness function $S=S(\theta, \phi)=$ $\left\{S_{j}, j=1, \ldots, N_{\mathrm{c}}\right\}$, for example, one has

$$
S(\theta, \phi)=\sum_{\ell=0}^{\infty} \sum_{m=-\ell}^{\ell} b_{\ell m} Y_{\ell m}(\theta, \phi) \Longrightarrow S_{\ell}=\frac{1}{2 \ell+1} \sum_{m}\left|b_{\ell m}\right|^{2},
$$

where $S_{\ell}$ is the corresponding angular power spectrum. Similar expressions hold for the $K=K(\theta, \phi)$.

Thus, to obtain quantitative information for each multipole component $\ell$ about the non-Gaussianity at large-angles (low $\ell$ ) from the $\mathbf{S}-$ and $\mathbf{K}$-maps, which were obtained from the CMB temperature maps, we calculate their power spectra $S_{\ell}$ and $K_{\ell}$. Finally, to compare the statistical properties of the spectra $\left\{S_{\ell}\right\}$ and $\left\{K_{\ell}\right\}$, obtained from the analyses of the different Monte Carlo CMB sets, we compare their corresponding mean $\bar{S}_{\ell}$ and $\bar{K}_{\ell}$ and 2 -sigma values (i.e., 95\% CL). Here we concentrate on the large angular scales, that is, for $\ell=1-10$.

\section{Results and Conclusions}

In this section we report the results of Gaussianity analyses performed by applying our indicators, discussed in the previous section, to the Monte Carlo and WMAP data described in Sec. 2. 
In Fig. 2 we show the APS of the $\mathbf{S}$-maps and $\mathbf{K}$-maps calculated from the Monte Carlo CMB maps, which were produced by the APS with slightly different spectral indexes, namely $n_{s}=0.96$ and 1.00. As expected, the similarity in the CMB APS implies in the similarity of the angular power spectra obtained from the $\mathbf{S}-$ and $\mathbf{K}$-maps. We also exhibit in these plots the APS from the $\mathbf{S}-$ and $\mathbf{K}$-maps obtained from the ILC-7yr WMAP map (with the corresponding mask to ensure Gaussianity properties, see Sec. 2).

Our main conclusion is that, as observed in Fig. 2, Monte Carlos generated through APS with a different spectral index $n_{s}$ have different statistical properties and therefore they show different confidence level when used to evaluate the statistical significance of some result obtained from WMAP data. For instance, the confidence level of the APS $\left\{\mathrm{S}_{\ell}\right\}$ and $\left\{\mathrm{K}_{\ell}\right\}$ for the WMAP ILC-7yr foregroundreduced map reveal that it is more likely non-Gaussian $\left(n_{s}=0.96\right)$ than Gaussian $\left(n_{s}=1.00\right)$. In order to quantify the possible correlation between data from WMAP and data from the sets of Monte Carlo maps we use the reduced $\chi^{2}$-goodness-offit test which gives an overall measure between two data sets by quantifying how related they are. For this we shall calculate the value $\chi^{2} / d o f \equiv \chi_{\text {red }}^{2}$, where $d o f$ stands for degree of freedom, between pairs of data sets regarding the APS values $\mathrm{S}_{\ell}$ and $\mathrm{K}_{\ell}$, for $\ell=1-10$, calculated from the ILC map versus the mean-APS $\overline{\mathrm{S}}_{\ell}$ and $\overline{\mathrm{K}}_{\ell}$ obtained from the two sets of Monte Carlo CMB maps, those produced with $n_{s}=0.96$ and $n_{s}=1.00$ (see Fig. 2).

Our results are: $\chi_{\text {red }}^{2}=1.8(1.3)$ between the APS of the $\mathbf{S}$-map $(\mathbf{K}$-map) from the ILC versus the mean APS of the $\mathbf{S}$-maps $(\mathbf{K}$-maps) obtained from Monte Carlo CMB maps with $n_{s}=0.96$. Similarly, we obtain $\chi_{\text {red }}^{2}=4.7$ (4.3) between the APS of the $\mathbf{S}-$ map $(\mathbf{K}-$ map) from the ILC versus the mean APS of the $\mathbf{S}$-maps $\left(\mathbf{K}\right.$-maps) obtained from Monte Carlo CMB maps with $n_{s}=1.00$. Remember that a $\chi^{2}$ value near 1 means a high correlation between analyzed data, while larger values means poorer correlations. Thus, our results indicate that is more probable that (the $\left\{\mathrm{S}_{\ell}\right\}$ and $\left\{\mathrm{K}_{\ell}\right\}$ APS from) the WMAP ILC map plus the KQ75-7yr mask corresponds to the case $n_{s}=0.96$, with a $p$-value of $\sim 15 \%$, than to the Gaussian case $n_{s}=1.00$, with a $p$-value of less than $0.1 \%$.

Summarizing, our APS analyses of $\mathbf{S}-$ and $\mathbf{K}$-maps produced from the two sets of Monte Carlo CMB maps (seeded by $\Lambda$ CDM spectra with $n_{s}=0.96,1.00$, respectively) show that, in the average, these data contain different amounts of nonGaussianity. Furthermore, Monte Carlos seeded on the $\Lambda$ CDM concordance model spectrum $\left(n_{s}=0.96\right)$ can not be considered as the absolute reference for Gaussian properties in CMB maps. Additionally, our results show that, in the average, the Gaussianity property of these sets of Monte Carlo maps is different, and this fact seems to be crucial when one has to quantify the confidence level of some result. That is to say, the statistical-significance evaluation of any result concerning WMAP data is model dependent.

It would be interesting to test the Gaussian properties of these two sets of Monte Carlo CMB maps using some statistical tools available in the literature. 

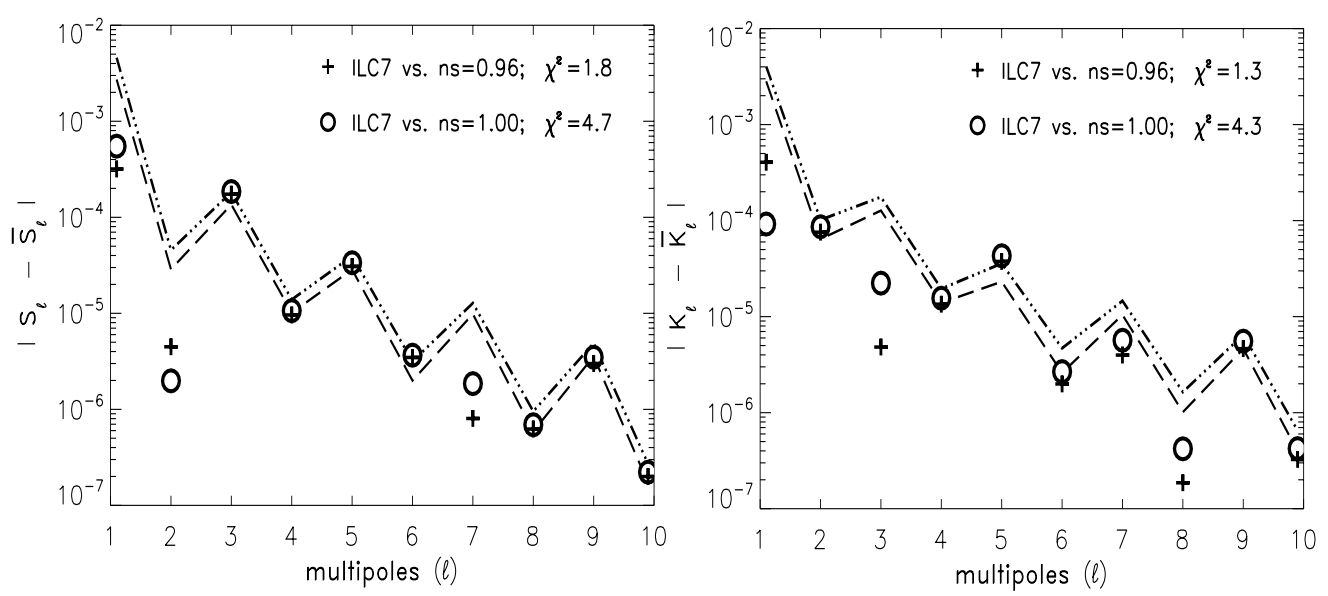

Fig. 2. Angular Power Spectra for the $\mathbf{S}-$ and $\mathbf{K}-$ maps, left and right panel, respectively. The dot-dashed (dashed) line corresponds to the $2 \sigma$ level (95\% confidence level) of the $\mathbf{S}-$ and $\mathbf{K}-$ map's power spectra, maps obtained by applying our statistical tools to 1000 Monte Carlo CMB maps produced from a $\Lambda$ CDM model with spectral index $n_{s}=0.96\left(n_{s}=1.00\right)$.

\section{Acknowledgments}

We acknowledge the use of the Legacy Archive for Microwave Background Data Analysis (LAMBDA). ${ }^{1}$ Some of the results in this paper were derived using the HEALPix package. ${ }^{23}$ This work was partially supported by the Fundação de Amparo à Pesquisa do Estado de Minas Gerais - Brasil (FAPEMIG) under grant APQ01893-10. A.B. also thanks the Brazilian agency CNPq for his grant.

\section{References}

1. N. Jarosik et al., Seven-year Wilkinson microwave anisotropy probe (WMAP) observations: Sky maps, systematic errors, and basic results, arXiv:1001.4744v1; D. Larson et al., Seven-year Wilkinson microwave anisotropy probe (WMAP) observations: Power spectra and WMAP-derived arameters, arXiv:1001.4635v1; http://lambda.gsfc.nasa.gov/

2. M. Cruz et al., New Astron. Rev. 50, 880 (2006); A. Bernui, C. Tsallis and T. Villela, Europhys. Lett. 78, 19001 (2007); Phys. Lett. A 356, 426 (2006); P. D. Naselsky et al., The mystery of the WMAP cold spot, arXiv:0712.1118v1; C. Monteserín et al., Mon. Not. R. Astron. Soc. 387, 209 (2008).

3. B. Lew, JCAP 08, 017 (2008); Y. Wiaux et al., Mon. Not. Roy. Astron. Soc. 385, 939 (2008); J. D. McEwen et al., Mon. Not. R. Astron. Soc. 388, 659 (2008); M. Kawasaki et al., JCAP 11, 019 (2008); C. Raeth et al., A model-independent test for scale-dependent non-Gaussianities in the CMB, arXiv:0810.3805v1.

4. G. Rossmanith, C. Raeth, A. J. Banday and G. Morfill, Non-Gaussian Signatures in the five-year WMAP data as identified with isotropic scaling indices, arXiv:0905.2854v1; P. Vielva and J. L. Sanz, Mon. Not. R. Astron. Soc. 397, 837 (2009); M. Kawasaki, K. Nakayama and F. Takahashi, JCAP 01, 026 (2009); M. 
Kawasaki et al., JCAP 01, 042 (2009); D. Pietrobon et al., Mon. Not. Roy. Astron. Soc. 396, 1682 (2009).

5. M. Cruz, E. Martínez-González and P. Vielva, The WMAP cold spot, arXiv:0901.1986v1; P. Cabella et al., Foreground influence on primordial nonGaussianity estimates: needlet analysis of WMAP 5-year data, arXiv:0910.4362 v1; A. Bernui and M. J. Rebouças, Int. J. Mod. Phys. A 24, 1664 (2009); Int. J. Mod. Phys. D 19, 1411 (2010).

6. D. Pietrobon et al., Mon. Not. Roy. Astron. Soc. 402, L34 (2010); Y. Ayaita, M. Weber and C. Wetterich, Phys. Rev. D 81, 023507 (2010); C. Raeth et al., Scaledependent non-Gaussianities in the WMAP data as identified by using surrogates and scaling indices, arXiv:1012.2985v1; A. P. S. Yadav and B. D. Wandelt, Primordial non-Gaussianity in the cosmic microwave background, arXiv:1006.0275v1.

7. C. Raeth et al., Probing non-Gaussianities on large scales in WMAP5 and WMAP7 data using surrogates, arXiv:1005.2481v1; N. Bartolo, S. Matarrese, O. Pantano and A. Riotto, Class. Quant. Grav. 27, 124009 (2010); N. Bartolo, S. Matarrese and A. Riotto, Non-Gaussianity and the cosmic microwave background anisotropies, arXiv:1001.3957; A. Bernui, M. J. Rebouças and A. F. F. Teixeira, Int. J. Mod. Phys. D 19, 1405 (2010); R. Saha, A foreground cleaned CMB map from non-Gaussianity measurement, arXiv:1105.6298v1.

8. J. D. McEwen, M. P. Hobson, A. N. Lasenby and D.J. Mortlock, Mon. Not. Roy. Astron. Soc. 371, L50 (2006); R. J. Adler, J. D. Bjorken and J. M. Overduin, Finite cosmology and a CMB cold spot, arXiv:gr-qc/0602102; L. R. Abramo et al., Phys. Rev. D 74, 063506 (2006); A. Bernui et al., Astron. Astrophys. 454, 409 (2006); P. Vielva, Y. Wiaux, E. Martínez-González and P. Vandergheynst, Mon. Not. Roy. Astron. Soc. 381, 932 (2007); A. Gruppuso et al., New constraints on paritysSymmetry from a re-analysis of the WMAP-7 low resolution power spectra, arXiv:1006.1979v1.

9. A. Gruppuso, Phys. Rev. D 76, 083010 (2007); T. Ghosh, A. Hajian and T. Souradeep, Phys. Rev. D 75, 083007 (2007); A. Bernui, B. Mota, M. J. Rebouças and R. Tavakol, Astron. Astrophys. 464, 479 (2007); Int. J. Mod. Phys. D 16, 411 (2007); C. Gordon, Astrophys. J. 656, 636 (2007).

10. B. Lew, JCAP 09, 023 (2008); P. K. Samal, R. Saha, P. Jain and J. P. Ralston, Mon. Not. R. Astron. Soc. 385, 1718 (2008); T. Koivisto and D. F. Mota, JCAP 06, 018 (2008); C. Monteserin et al., Mon. Not. R. Astron. Soc. 387, 209 (2008); T. Kahniashvili, G. Lavrelashvili and B. Ratra, Phys. Rev. D 78, 063012 (2008).

11. A. Bernui and W. S. Hipólito-Ricaldi, Mon. Not. R. Astron. Soc. 389, 1453 (2008); P. Vielva and J. L. Sanz, Analysis of non-Gaussian CMB maps based on the N-pdf: Application to WMAP data, arXiv:0812.1756v1; A. Bernui, Phys. Rev. D 78, 063531 (2008); P. K. Samal, R. Saha, P. Jain and J. P. Ralston, Mon. Not. R. Astron. Soc. 396, 511 (2009); C. M. Hirata, JCAP 09, 011 (2009).

12. C. Caprini, F. Finelli, D. Paoletti and A. Riotto, JCAP 06, 021 (2009); J. Kim and P. Naselsky, JCAP 07, 041 (2009); I. Y. Areféva, N. V. Bulatov, L. V. Joukovskaya and S. Y. Vernov, Phys. Rev. D 80, 083532 (2009); 80, 123010 (2009); Y. Shtanov and H. Pyatkovska, Phys. Rev. D 80, 023521 (2009); G. P. Holder, K. M. Nollett and A. van Engelen, On possible variation in the cosmological baryon fraction, arXiv:0907.3919v1.

13. L. R. Abramo, A. Bernui and T. S. Pereira, JCAP 12, 013 (2009); A. L. Erickcek, C. M. Hirata and M. Kamionkowski, Phys. Rev. D 80, 083507 (2009); J. F. Donoghue, K. Dutta and A. Ross, Phys. Rev. D 80, 023526 (2009); N. Joshi, S. Jhingan, T. Souradeep and A. Hajian, Bipolar harmonic encoding of CMB correlation patterns, arXiv:0912.3217v1; T. R. Seshadri and K. Subramanian, Phys. Rev. Lett. 103, 081303 (2009). 
14. T.-P. Li, H. Liu, L.-M. Song, S.-L. Xiong and J.-Y. Nie, Observation number correlation in WMAP data, arXiv:0905.0075v1; M. Frommert and T. A. Ensslin, The axis of evil - a polarization perspective, arXiv:0908.0453v1; L. R. Abramo and T. S. Pereira, Testing gaussianity, homogeneity and isotropy with the cosmic microwave background, arXiv:1002.3173v1; H.-C. Kim and M. Minamitsuji, Scalar field in the anisotropic universe, arXiv:1002.1361v1.

15. A. Gruppuso and K. M. Górski, JCAP 03, 019 (2010); D. G. Yamazaki, K. Ichiki, T. Kajino and G. J. Mathews, Phys. Rev. D 81, 023008 (2010); A. R. Pullen and C. M. Hirata, Non-detection of a statistically anisotropic power spectrum in largescale structure, arXiv:1003.0673v1; S.-Y. Zhong and X. Wu, Phys. Rev. D 81, 104037 (2010); S.-Y. Zhong, X. Wu, S.-Q. Liu and X.-F. Deng, Phys. Rev. D 82, 124040 (2010).

16. M. Cruz, P. Vielva, E. Martínez-González and R. B. Barreiro, Anomalous variance in the WMAP data and galactic foreground residuals, arXiv:1005.1264v1; F. Paci et al., Mon. Not. Roy. Astron. Soc. 407, 399 (2010); T. Kahniashvili, A. Brandenburg, A. G. Tevzadze and B. Ratra, Phys. Rev. D 81, 123002 (2010); D. Pietrobon, A. Balbi, P. Cabella and K. M. Górski, Astrophys. J. 723, 1 (2010); R.-G. Cai, B. Hu and H.-B. Zhang, JCAP 08, 025 (2010).

17. F. R. Urban and A. R. Zhitnitsky, The P-parity odd universe, dark energy and QCD, arXiv:1011.2425v1; P. K. Aluri, P. K. Samal, P. Jain and J. P. Ralston, Effect of foregrounds on the CMBR multipole alignment, arXiv:1007.1827v1; C. J. Copi, D. Huterer, D. J. Schwarz and G. D. Starkman, Adv. Astron. 2010, 847541 (2010); I. Ya. Areféva, N. V. Bulatov and S. Yu. Vernov, Theor. Math. Phys. 163, 788 (2010); N. Bartolo, M. Fasiello, S. Matarrese and A. Riotto, JCAP 12, 026 (2010).

18. H.-C. Kim and M. Minamitsuji, Phys. Rev. D 81, 083517 (2010); 82, 109904 (2010); JCAP 03, 038 (2011); H. Wei, JCAP 04, 022 (2011); N. Afshordi, A. Slosar and Y. Wang, JCAP 01, 019 (2011); J.-Q. Xia et al., Constraints on primordial nonGaussianity from large scale structure probes, arXiv:1104.5015v1.

19. N. Bartolo et al., Phys. Rep. 402, 103 (2004); E. Komatsu et al., Non-Gaussianity as a probe of the physics of the primordial universe and the astrophysics of the low redshift universe, arXiv:0902.4759v1.

20. A. Bernui and M. J. Rebouças, Phys. Rev. D 79, 063528 (2009); 81, 063533 (2010).

21. F. Elsner and B. D. Wandelt, Astrophys. J. Suppl. Ser. 184, 264 (2009).

22. http://lambda.gsfc.nasa.gov/toolbox/tb_cmbfast_ov.cfm, http://lambda.gsfc.nasa.gov/toolbox/tb_camb_form.cfm

23. K. M. Górski et al., Astrophys. J. 622, 759 (2005). 